\title{
LOS PROCESOS FONOLÓGICOS DE ELISIÓN Y SUSTITUCIÓN EN EL CORPUS PRESEEA-MEDELLÍN ${ }^{1}$ \\ PHONOLOGICAL PROCESSES OF ELISION AND SUBSTITUTION IN THE CORPUS PRESEEA-MEDELLIN
}

\author{
María Claudia González-Rátiva \\ Universidad de Antioquia
}

Este artículo presenta el análisis variacionista de los procesos fonológicos de elisión y sustitución presentes en 36 entrevistas semidirigidas del Corpus Preseea-Medellín. Se comprueba la hipótesis de que los procesos fonológicos están condicionados por factores internos a través del análisis de regresión múltiple realizado con el programa estadístico GoldVarb. Los resultados mostraron que la aplicación de los procesos fonológicos de elisión y sustitución guardan una relación multicausal, especialmente con factores de tipo semántico como la funcionalidad y la homofonía y aspectos de nivel fonológico como el entorno segmental de palabra, el número de sílabas, la homorganicidad y el acento. La interpretación de los datos da cuenta de que las variables internas son las que guían el procesamiento del lenguaje para la estructuración léxica en la posibilidad variable de elidir o sustituir una posición fonológica en la cadena fónica. Estos patrones responden a restricciones del orden de la transparencia semántica y la evasión de la similitud en mayor medida para colaborar con la comprensión del mensaje. En este sentido, la variedad medellinense de habla coopera con la comprensión del mensaje en el uso de la lengua.

Palabras Clave: procesos fonológicos, español hablado en Medellín, corpus PreseeaMedellín, análisis de regla variable

This article presents the variationist analysis of phonological processes of elision and substitution present in 36 semi-structured interviews Corpus Preseea-Medellin. The hypothesis that phonological processes are conditioned by internal factors through multiple regression analysis performed with the GoldVarb statistical program is checked. The results showed that the application of phonological processes of elision and substitution has a multi-causal relationship, especially between semantic factors as functionality and homophony, and aspects of phonology as the segmental environment of speech, number of syllables, the homorganicity and accent. The interpretation of the data accounts that the internal variables may guide language processing to conform lexical structure where there is the possibility to elide or replace a phonemic position in the phonic chain. These patterns respond to restric-

\footnotetext{
${ }^{1}$ Este artículo es parte de la tesis doctoral “Análisis sociolingüístico de los procesos fonológicos en el habla de Medellín”, cuya dirección estuvo a cargo de la doctora Marianne Dieck, de la Universidad de Antioquia, y con la codirección de la doctora Luz Marcela Hurtado Cubillos, de Central Michigan University.
} 
tions on the order of semantic transparency and avoidance of similarity to a greater extent to assist with understanding the message. In this sense, the speakers of Spanish spoken in Medellin cooperate with comprehension of the messages in language use.

KEYwORDS: phonological processes, Spanish spoken in Medellín, Preseea-Medellín corpus, variable rule analysis

RECEPCIÓN: 03/08/2016

ACEPTACión: 29/09/2016

\section{Introducción}

En el habla se presentan comúnmente formas léxicas que en su realización no siguen los modelos canónicos de pronunciación del léxico de la lengua. Estudios sobre el cambio lingüístico, la adquisición, la variación o aquellos que implican tareas cognitivas del componente fonológico en la psicolingüística y la neurolingüística tratan estos fenómenos bajo el rótulo de procesos fonológicos y los relacionan causalmente con analogías, efectos coarticulatorios o variación estilística y social. Estas divergencias de la estructura léxica "pueden ser eventos en la historia de una lengua o relaciones entre la representación fonológica más abstracta de un segmento o grupo de segmentos y su realización fonética superficial" (Hulst, 2001: 3).

Algunas teorías del procesamiento léxico del lenguaje (Juncos Rabadán, Elosúa de Juan, Pereiro Rozas y Torres Maroño, 1998: 170) y la producción de una palabra en el habla (Levelt, 2001; Levelt, Roelofs y Meyer, 1999) dan cuenta de cómo son las representaciones mentales, de qué manera el ser humano almacena la información, cómo está organizado el lexicón mental y cómo se recupera esa información para hacerla transmisible y exitosa en la comunicación. De acuerdo con Levelt (1999: 223), el lexicón mental contiene representaciones de diversos tipos, incluidas las fonológicas y las fonéticas (a través del gesto articulatorio). De acuerdo con estas teorías, en el lexicón mental operan dos mecanismos, el de activación-inhibición (se activa la unidad adecuada y se inhiben las menos óptimas) y el de trans- 
misión, que conecta los niveles con la opción adecuada (Denes, Semenza y Magno Caldognetto, 1999: 197). La forma codificada es el producto final del acceso léxico (Levelt, 2001: 13465), resultado de la codificación fonológica que es una representación abstracta también, conocida como la planeación fonética (Denes et al., 1999: 199).

Vemos entonces cómo la unidad de representación fonológica, el fonema, es una unidad de procesamiento léxico presente e importante en cada etapa del proceso lingüístico, desde el almacenamiento hasta la planeación. El fonema, como una de las unidades de procesamiento léxico, tiene un lugar relevante en cada etapa. La posición fonológica que ocupa cada segmento, incluyendo el fonema cero (Jakobson, 1939, citado en Battistella, 1996: 23), que forma parte del sistema fonológico de la lengua, desempeña la función de contribuir con la generación del lexema significante, desde su almacenamiento y retroalimentación constante como forma, en la memoria fonológica, en la competencia de formas, en la activación o inhibición de patrones, en la silabificación y en la creación de un patrón articulatorio. Incluso, desde un punto de vista neuropsicológico, algunos estudios indican que cuando un paciente no es capaz de producir una palabra, el efecto "lo tengo en la punta de la lengua" significa que guarda cierta información sobre el fonema inicial, número de sílabas, ritmo, lugar del acento, por ejemplo, que puede implicar que el lemma, unidad de entrada al lexicón ya está construido morfonológicamente y que se alcanza información parcial (Denes et al., 1999: 206). ${ }^{2}$

Ahora bien, los modelos de análisis cuantitativos que buscan patrones internos, externos y cognitivos a través de pruebas estadísticas de regresión logística y cálculos de probabilidades nos permiten explicar el uso de diversas formas que compiten

\footnotetext{
${ }^{2}$ Sin embargo, también advierten estos autores la importancia de distinguir entre falta de acceso al lexicón fonológico o problemas de representación y una transmisión pobre o una planeación deficiente de la articulación. El estudio del procesamiento cognitivo del léxico se ha realizado, principalmente, a través de errores de habla en pacientes afásicos, en aprendices de lengua extranjera y en el habla cotidiana.
} 
en el habla y entender un poco mejor la gramática de la comunidad. De esta manera, se integra una visión sociolingüística cognitiva y cuantitativa al tratamiento de la variación fonológica y se llega a una explicación multicausal más cercana a la competencia sociolingüística de la comunidad (Chambers, Trudgill y Schilling-Estes, 2004: 118).

En este trabajo, el proceso fonológico o posición fonológica elidida o sustituida sobre el sistema fónico del español en la representación léxica se pone como fenómeno en estudio. Se presentan a continuación los resultados del estudio variacionista de los procesos fonológicos de elisión y sustitución en el habla de Medellín. Se parte de la hipótesis de que los procesos fonológicos de elisión y sustitución están condicionados por factores internos y externos. De esta manera, se asume la variación sociolingüística de los procesos fonológicos como opciones que tienen los hablantes para modificar los patrones léxicos y cómo se constituye una norma social a partir de esa variación. De acuerdo con Labov, "Muchas explicaciones del cambio lingüístico se apoyan marcadamente en el principio del menor esfuerzo y suponen sin justificación que el cambio conduce hacia la simplificación [...] Los factores sociales que favorecen e inhiben estos cambios son incluso de mayor interés" (1996: 11). De ahí el empeño de este trabajo por correlacionar los factores lingüísticos, estilísticos y sociales como variables independientes, que puedan estar afectando una variable dependiente como el grupo de procesos fonológicos que modifican las formas lexemáticas del habla en la comunidad de Medellín. Dos preguntas guiaron la investigación: ¿Qué aspectos, entre los gramaticales, estilísticos y sociales, favorecen la aparición de procesos fonológicos en el habla de Medellín? ¿Qué inferencias explicativas se pueden derivar de la significatividad estadística?

\section{Metodología}

El objetivo del trabajo consistió en analizar cuantitativamente los procesos fonológicos de elisión y sustitución fonemática presentes en las entrevistas semidirigidas de 36 informantes del 
Corpus Preseea-Medellín (González-Rátiva, 2008), ${ }^{3}$ a partir de su interacción con factores internos y externos, con el fin de encontrar tendencias sistémicas, reestructuraciones sistemáticas, que puedan dar cuenta de la conciencia sociolingüística y su pertinencia, de la norma de la comunidad. De esta manera, las conclusiones pretenden aportar a una teoría de los procesos fonológicos en el marco mismo del sistema lingüístico, vacío teórico señalado en relación con las teorías fonológicas (Anderson, 1980; Varela Ortega, 1984); además, contribuyen al estudio de la variedad de habla de la comunidad, del español hablado en Colombia y a la lingüística histórica, en general.

La distribución de los informantes fue la siguiente: de acuerdo con las características de preestratificación por cuotas de muestreo: 18 mujeres y 18 hombres; 12 informantes de cada generación o grupo etario (20-34, 35-54, 55- ); 11 individuos con nivel educativo bajo, 11 con nivel educativo medio y 14 de nivel alto; y de acuerdo con la posestratificación por clase social: ${ }^{4} 18$ informantes de clase popular, 11 de clase media y 7

\footnotetext{
${ }^{3}$ La metodología se sigue a partir de los parámetros del Proyecto para el Estudio Sociolingüístico del Español de España y América (PRESEEA, 2003), adaptados para su aplicación a la comunidad de Medellín (Andrade Rodríguez, González Rátiva y Jaramillo Yepes, 2008) en sus condiciones sociales de preestratificación y posestratificación (González Rátiva y Grajales Alzate, 2011). El Corpus PreseeaMedellín está alojado en un portal web de la Facultad de Comunicaciones de la Universidad de Antioquia, contiene 119 audios y transliteraciones de entrevistas semidirigidas y puede ser consultado y utilizado para investigación mediante registro e inscripción en http://comunicaciones.udea.edu.co/corpuslinguistico/

${ }^{4}$ La postestratificación en clase social se realizó para el Preseea-Medellín con base en una ponderación de factores sociales, características de estilos de vida y datos de nivel socioeconómico (González Rátiva y Grajales Alzate, 2011). A partir de la reflexión crítica sobre el concepto 'nivel sociocultural' y con base en la propuesta de Macionis y Plummer (1999) sobre la configuración de las clases sociales en una comunidad, se tomaron varios parámetros de la ficha de datos de cada informante, entre ellos, los ingresos individuales y familiares, la posesión de bienes inmuebles, la estratificación socioeconómica, el nivel educativo y la ocupación, contextualizados en nuestra sociedad, para generar un modelo de escala mixta de ponderación que estableció a qué clase social pertenecía cada uno de los informantes de la muestra. Se estableció que los informantes del Preseea-Medellín "pueden ajustarse como pertenecientes a la clase media (media-alta, media y media-baja) y a la clase trabajadora de la teoría mencionada” (González Rátiva y Grajales Alzate, 2011:
} 
de clase media alta. Se identificó, codificó y sistematizó como fenómeno variable el proceso fonológico o posición fonológica elidida o sustituida a partir de varios registros auditivos. ${ }^{5}$

La variable dependiente en este estudio agrupó la variación fonética en casos de procesos fonológicos de elisión o sustitución en cualquier posición de la cadena fónica. Los procesos fonológicos seleccionados se basan en la ausencia (no realización) de un segmento fónico esperado y en la permutación de un fonema por otro (realización articulatoria no predicha por las reglas de la gramática en la posición de otra), lo que hace a estos fenómenos evidentes impresionísticamente. Se seleccionaron para llevar a análisis un total de 9,597 casos o tokens de elisiones y sustituciones. El cuadro 1 muestra la distribución de la muestra de procesos fonológicos para este estudio.

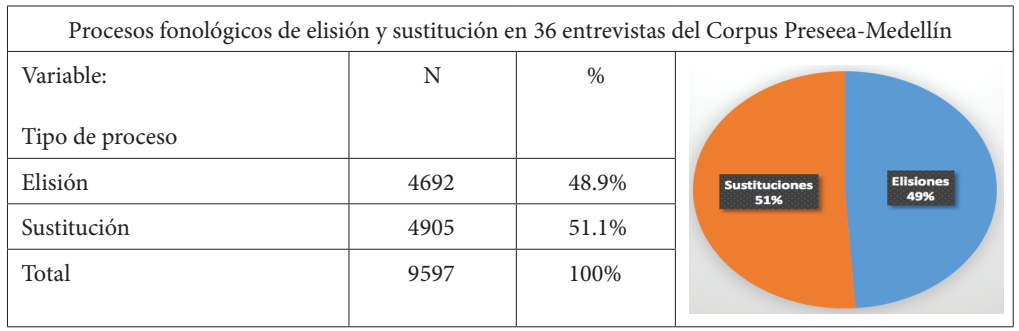

Cuadro 1. Frecuencias absolutas de los casos de procesos fonológicos

Como variable dependiente, el grupo de elisiones y sustituciones involucró cada uno de los segmentos fonológicos de la lengua y su relación con el contexto, la sílaba, el acento y otros aspectos que pueden llegar a afectar su realización en el habla se incluyeron en el estudio como variables independientes.

60). La nominación como popular (trabajadora), media (media-baja y media) y media alta responde a la percepción de la comunidad, expresada en una de las respuestas del cuestionario.

${ }^{5}$ La audición se hizo con auriculares Sennheisser HD201 y de acuerdo con la calidad de la grabación se realizaron múltiples audiciones por fragmento. Una vez percibido un fenómeno y, en caso de requerirlo, se corroboró a través de registro espectrográfico mediante Praat (Boersma y Weenink, 2014), se resaltó en el archivo de texto y se adscribió bajo un código en una plantilla de cuantificación del programa Excel. 
En relación con las elisiones, algunas veces ya estaban incorporadas en la transliteración ortográfica por medio de la etiqueta $<$ sic $>$; pero en la mayoría de los casos no fueron señaladas en el trabajo de etiquetación. De los 4,692 casos de elisión, la mayoría (90.27\%) corresponden a elisiones de los fonemas /d/ (44.5\%), /s/ (27.7\%), /f/ (9.36\%), /k/ 6.31\%) y /n/ (2.4\%), en ejemplos como [meØe'dzin] por /mede' $\lambda$ in/ (MEDE_

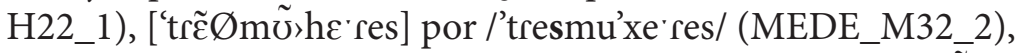
[dibećn'ti Øse] por /diber'tirse] (MEDE_M11_2), [direØ'sjõ'n] por/direk' sion/ (MEDE_H21_2) y [en_to' Oses] por / [en'tonses] (MEDE_M32_1).

Para el caso de las sustituciones, se percibieron y codificaron como tal 4905 casos que en su mayoría (98.28\%) corresponden a fenómenos asociados con el yeísmo (49.9\%), la desfonologización de las vibrantes (30.3\%), la debucalización o aspiración de la /s/ (13.9\%) y de la /f/ (3.2\%) y los casos de sustitución de vocales medias por vocales altas (0.98\%) /e/ /o/ $\rightarrow$ [i] [u]. Son ejemplos correspondientes de las diferentes sustituciones: [ka'bejo] por /ka'beאo/ (MEDE_M22_1), [ [ $\left.\int a r^{\prime} l a r\right]$ por / ţar'lar/ (MEDE_H22_1), [es'țã 'mõhespe'rã'ndo] por / es'tamosespe'rando/ (MEDE_H12_4), [ĩnõØ'hwĩmõs] por / inos'fui'mos] (MEDE_H21_4), [dispeç'te'] por /desper'te/ (MEDE_M21_2). Obsérvese que para el caso de la palatal lateral $/ \Lambda /$, aunque se ha establecido la norma yeísta para esta zona del país, el abanico de variación por sustitución para este segmento no solo incluye casos de desfonologización en /j/ sino sus pronunciaciones como semiconsonante, africada, o como vocal.

Como variables independientes ${ }^{6}$ se determinaron y definieron 15 grupos factoriales lingüísticos (posición del acento, número de sílabas, estructura acentual, posición silábica, contexto

\footnotetext{
${ }^{6}$ Para el establecimiento de los factores lingüísticos y extralingüísticos, se partió de la propuesta de codificación para el estudio de la elisión de /d/ en participios ado, realizada en el marco del PRESEEA, expuesta en el "Proyecto coordinado Estudio sociolingüístico del español de Las Palmas, Lleida, Granada, Madrid-Alcalá, Málaga, Sevilla y Valencia” (Gómez Molina, Ávila y Vida, 2007) y coordinada por J. A. Samper Padilla ya que presenta un grupo muy completo de aspectos contextuales, estilísticos y sociales.
} 
segmental, homorganicidad, entorno segmental de palabra, entorno de grupo fónico, turno anterior, tipo de vocabulario, categoría léxica, homofonía, funcionalidad, ${ }^{7}$ estructura morfemática, difusión léxica); un grupo factorial estilístico (inicio, parte media, parte final de la entrevista); y cuatro grupos factoriales sociales (sexo, edad, nivel educativo y clase social). Es importante aclarar que las entrevistas semidirigidas están organizadas en un continuum temporal-estilístico que permite ubicar fragmentos de modos discursivos orales que bien pueden definirse como: modo descriptivo en estilo (+) formal, modo explicativo argumentativo en estilo (+/-) formal y un modo narrativo cuyo estilo se acerca al registro (+) coloquial. Este continuum temporal estilístico se sustenta de igual forma con una visión dinámica o gradual de la interacción en la entrevista (Briz, 2010: 128), según la cual, dados los rasgos del evento comunicativo, hay un proceso que se va cambiando o moviendo de un registro (+) formal en los primeros momentos o al inicio de la entrevista, pasa por un registro (+/-) formal en su parte media y finaliza en un registro (+/-) coloquial.

En total se determinaron 54 factores de análisis como variables independientes que podrían estar favoreciendo o no la aplicación de un proceso fonológico de elisión o sustitución. De esa manera, la codificación de los 9,597 casos de elisiones y sustituciones, no solo se trató de la asignación realización-tipo, sino que en la relación proceso fonológico-factor lingüístico se requirió de análisis lingüístico y de revisión de muchos de los casos de este trabajo, lo que aleja el carácter subjetivo de la codificación y reitera el valor metodológico del trabajo cualitativo en la sociolingüística. Para el análisis cuantitativo estadístico se uti-

\footnotetext{
${ }^{7}$ Funcionalidad se refiere a que la presencia del fenómeno puede verse inhibida porque se genera con la variación una ambigüedad o confusión semántica, a pesar de la existencia de otras condiciones que favorecen la variabilidad. Tres factores: no hay ambigüedad funcionalidad virtual en una palabra como acosta(r)nos; se genera con el proceso fonológico un par funcional de distinta categoría léxica en el caso de e(s)e>eje (determinante/nombre); y cuando aparece el fenómeno se crea en el habla un par funcional de la misma categoría léxica, como en vi(d)a>vía (nombre). Se codificaron los casos en su relación con cada uno de estos tres factores o tipos de relación léxico-semántica.
} 
lizó el Programa Goldvarb (Sankoff, Tagliamonte y Smith, 2005) en su versión $3.0 \mathrm{~b} 8,{ }^{8}$ que ofrece las tres líneas de evidencia del análisis multivariado y permite obtener la ordenación de mayor a menor de las variables independientes estadísticamente significativas. La evidencia estadística de probabilidades permitió inferir resultados sobre los usos y preferencias fonológicas de la comunidad de habla de Medellín.

\section{Resultados}

El procedimiento de afinación del modelaje estadístico ${ }^{9}$ a través del programa Goldvarb se realizó a través de reagrupación, refactorización, chequeo y 7 cálculos de análisis de regresión múltiple de subida y bajada (up $\mathcal{E}$ down). En el quinto cómputo se separaron factores externos e internos y en el sexto cálculo se intercambiaron de orden de ingreso las variantes de la variable dependiente. No se encontró relevancia en la separación ni en el cambio de ingreso de datos; el orden del alcance de los grupos factoriales coincide mayoritariamente en los cálculos realizados. En el séptimo cómputo, el programa realizó análisis de regresión de subida y bajada en 21 niveles y 230 cómputos. Para la interpretación de los datos se tomaron los mejores cómputos (\#121 y \#122), que coincidieron en la selección de los grupos, por lo cual

\footnotetext{
${ }^{8}$ El programa hace un análisis de regresión múltiple y ofrece un cálculo de probabilidades. El programa de regresión múltiple realiza al mismo tiempo análisis y comparación de todas y cada una de las variables en estudio, para poder determinar cómo afectan a la variable dependiente. A través de los diferentes cómputos del análisis multivariado en el programa Goldvarb se presenta la relevancia estadística del efecto (significance), el peso de cada factor, el alcance del efecto (range) y la jerarquía de la influencia dada por el orden de los pesos en cada grupo factorial (Hurtado Cubillos, 2012: 150; Tagliamonte, 2006: 235).

${ }^{9}$ El procedimiento de afinación consiste en reagrupar, recodificar o eliminar factores. Se hace una constante revisión de algunos casos que no tengan relevancia estadística o que se relacionan con fenómenos morfosintácticos específicos o léxicos, rarezas lingüísticas o infrecuencias relativas. Toda esa información queda consignada y puede formar parte de la interpretación o de la explicación de otros fenómenos, o desde la perspectiva de la investigación, se reservan para nuevos estudios (Tagliamonte, 2006: 98).
} 
es un análisis fiable y de donde se tomaron los pesos factoriales para la construcción de los cuadros. La significancia del mejor cómputo fue de 0.032 , lo que indica que el análisis es estadísticamente significativo. De esta manera se rechaza la hipótesis nula, que establece que no hay condicionamientos que influyan para la aplicación de la regla variable.

En el séptimo cálculo, luego del análisis de subida y bajada, se realizó el análisis binomial de un paso que nos arroja la síntesis del análisis estadístico. Con esos datos se construyó el cuadro 2: grupos factoriales ordenados por alcance (range), que muestran la jerarquía de las restricciones, el orden de influencia de las variables independientes sobre la variable dependiente. Además, se destacan los pesos GoldVarb relevantes para la interpretación de la aplicación de las reglas de elisión y sustitución.

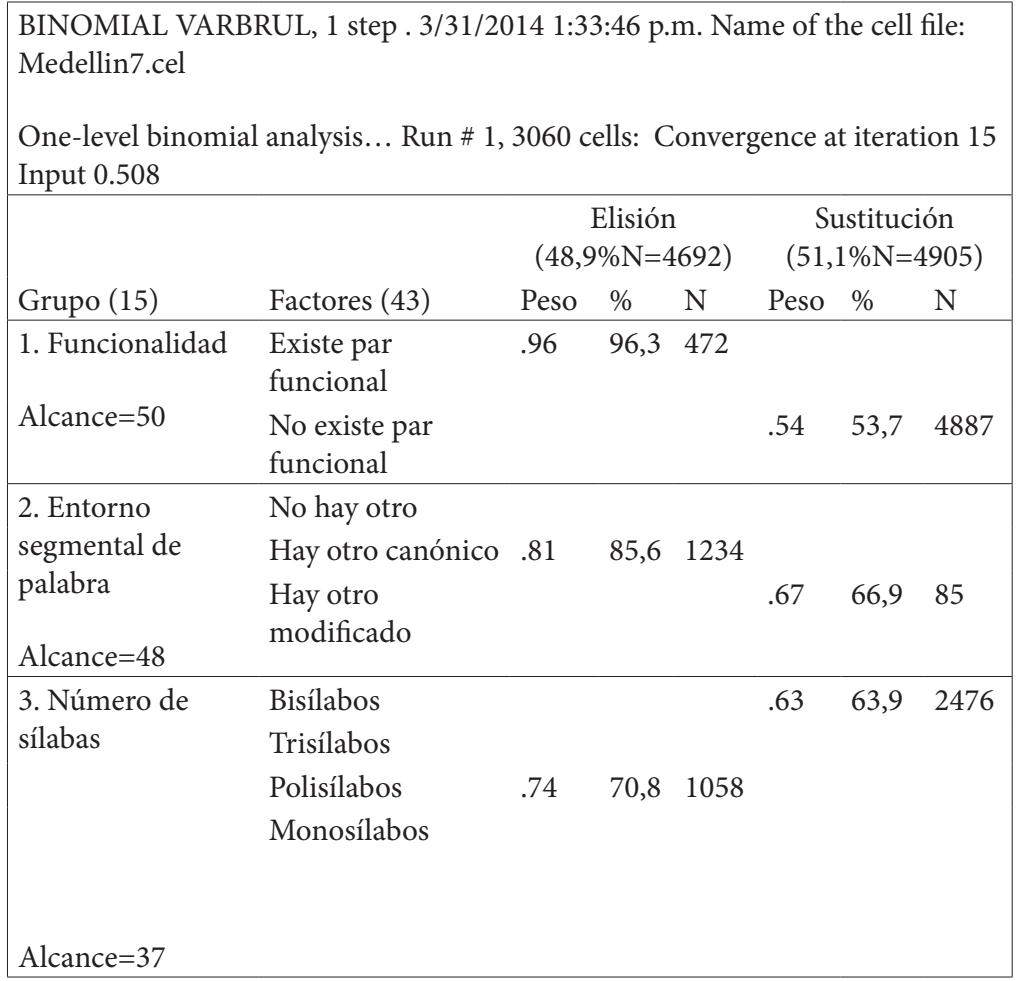




\begin{tabular}{|c|c|c|c|c|c|c|c|}
\hline 4. Homofonía & $\begin{array}{l}\text { Ausencia } \\
\text { Homofonía } \\
\text { (N,V,Adj.) }\end{array}$ & & & & .58 & 59,3 & 4178 \\
\hline Alcance $=35$ & $\begin{array}{l}\text { Homofonía } \\
\text { (Otras) }\end{array}$ & .77 & 79,9 & 1359 & & & \\
\hline 5. & Sin homorgánicos & .62 & 61,6 & 3599 & & & \\
\hline $\begin{array}{l}\text { Homorganicidad } \\
\text { Alcance }=30\end{array}$ & $\begin{array}{l}\text { Con } \\
\text { homorgánicos }\end{array}$ & & & & .68 & 70,9 & 2657 \\
\hline 6. Acento & $\begin{array}{l}\text { Precede } \\
\text { Sigue } \\
\text { Tónica }\end{array}$ & .70 & 57,9 & 1546 & .59 & 84,4 & 844 \\
\hline Alcance $=28$ & & & & & & & \\
\hline $\begin{array}{l}\text { 7. Contexto } \\
\text { segmental }\end{array}$ & $\begin{array}{l}\text { Ante vocal } \\
\text { Ante consonante } \\
\text { Ante pausa } \\
\text { Después de pausa }\end{array}$ & .60 & 59,1 & 2175 & .66 & 57,8 & 189 \\
\hline Alcance $=26$ & & & & & & & \\
\hline 8. Turno & $\begin{array}{l}\text { No se presenta } \\
\text { Hay canónicos } \\
\text { Hay modificados }\end{array}$ & .67 & 72.4 & 155 & .58 & 60,4 & 255 \\
\hline Alcance $=25$ & & & & & & & \\
\hline $\begin{array}{l}\text { 9. Entorno grupo } \\
\text { fónico } \\
\text { Alcance }=22\end{array}$ & $\begin{array}{l}\text { Hay canónico } \\
\text { No hay otro } \\
\text { Hay modificado }\end{array}$ & .63 & 72,6 & 2705 & .59 & 69,8 & 3006 \\
\hline $\begin{array}{l}\text { 10. Difusión } \\
\text { léxica Alcance=21 }\end{array}$ & $\begin{array}{l}\text { Alta } \\
\text { Baja }\end{array}$ & .66 & 53,9 & 1213 & .55 & 52,6 & 3867 \\
\hline $\begin{array}{l}\text { 11. Posición } \\
\text { silábica } \\
\text { Alcance }=18\end{array}$ & $\begin{array}{l}\text { Coda } \\
\text { Ataque }\end{array}$ & .52 & 40,2 & 1968 & .51 & 43,1 & 1920 \\
\hline $\begin{array}{l}\text { 12. Estructura } \\
\text { acentual }\end{array}$ & $\begin{array}{l}\text { Paroxítona } \\
\text { Oxítona } \\
\text { Proparoxítona }\end{array}$ & .63 & 76,8 & 116 & .51 & 50,2 & 2799 \\
\hline Alcance $=14$ & & & & & & & \\
\hline $\begin{array}{l}\text { 13. Estructura } \\
\text { morfemática } \\
\text { Alcance }=10\end{array}$ & $\begin{array}{l}\text { Afijo } \\
\text { Raíz }\end{array}$ & .58 & 65,8 & 1286 & .52 & 55,4 & 4237 \\
\hline
\end{tabular}




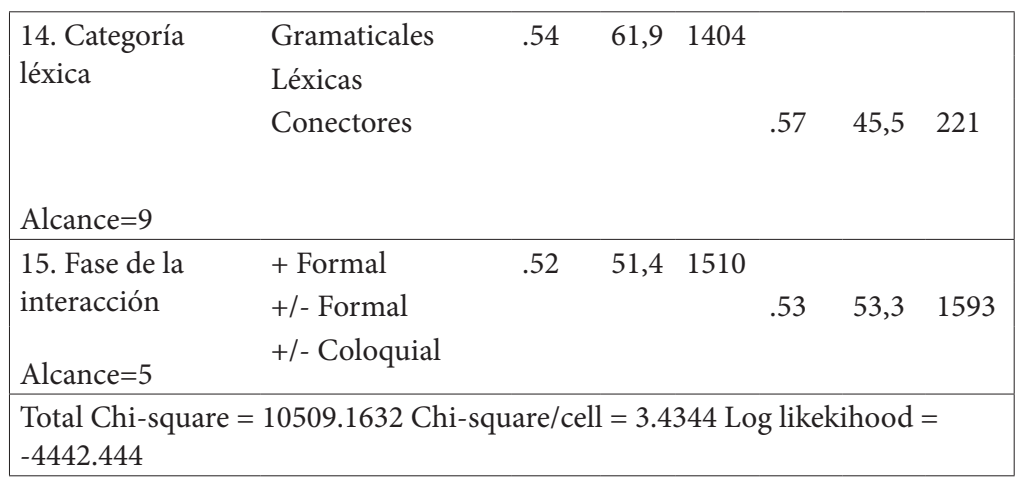

Cuadro 2. Orden de influencia y factores significativos para la aplicación de la regla de elisión y sustitución

El cuadro 2 muestra que todos los grupos factoriales internos fueron significativos y se destaca la ausencia de los factores sociales: en todos los cómputos de regresión múltiple, estos obtuvieron alcances muy bajos y, además, en varios de los cómputos se eliminó el factor edad como variable significativa.

La preponderancia de los factores internos sobre los factores sociales da cuenta de la importancia que revisten para los fenómenos de elisión y sustitución fonológica aquellos factores que son estructural o sistemáticamente motivados (Labov, 2006: 33). Que el hablante elida o sustituya una posición fonológica es principalmente una influencia ponderada de componentes del sistema lingüístico.

Se observa que los grupos que más influyen sobre las probabilidades de elidir y sustituir representaciones fonológicas son la funcionalidad (alcance 50), cuya variable de existencia de un par funcional en el léxico favorece el proceso de elisión, mientras la no existencia de ese par coadyuva a las sustituciones; y el entorno segmental de palabra (alcance 48), para cuyo caso la elisión se favorece cuando hay presencia de otro fonema canónico en el contexto léxico, mientras que cuando hay otro fonema modificado, se beneficia la probabilidad de sustituir fonemas. También contribuyen con el proceso de elisión las palabras polisilábicas, la existencia de homófonos de categoría distinta a la de la forma léxica que contiene el proceso y la presencia de fone- 
mas no homorgánicos en adyacencia. El proceso de sustitución se ve respaldado por los bisílabos, la ausencia de homófonos y el contacto con fonemas homorgánicos. De esta forma puede seguirse la información de resultados en el cuadro 2 hasta llegar al factor con menos alcance entre los factores internos, la fase de interacción o factor estilístico.

\section{Análisis y discusión de los resultados}

Los datos estadísticos nos permiten acercarnos a una interpretación más objetiva de los fenómenos. Los hechos y evidencias que se presentan pretenden encontrar sus causas en principios que, en términos generales, se esbozan por modelos y teorías sobre el lenguaje. Principalmente, se recurrirá en la interpretación a la Teoría de la producción del habla (Goldrick, 2014) y a la Teoría de la producción del habla y del cambio fónico (Garrett y Johnson, 2013).

Los procesos de elisión y sustitución realizados por el grupo de hablantes de la comunidad de Medellín constituyen una muestra representativa de la manera en que la gramática específica de esta comunidad organiza y re-organiza las posiciones fonológicas de los patrones léxicos de entrada; en términos de la sociolingüística Laboviana, estamos ante la re-intepretación de las posiciones fonológicas en esta comunidad. La influencia que ejercen otros elementos de la gramática y aquellos extralingüísticos sobre los procesos de elisión y sustitución forma parte de la gramática interna de la comunidad y de la competencia de sus hablantes.

\section{El efecto de los factores internos sobre la elisión y la sustitución}

De acuerdo con el Análisis de Regla Variable, cada factor correlacionado con los procesos fonológicos de elisión y sustitución se presenta como respuesta a la hipótesis de trabajo planteada. 
A continuación se presentan las inferencias y explicaciones de los factores internos con mayor peso significativo y las correlaciones pertinentes.

\section{Efecto de la funcionalidad}

\begin{tabular}{|c|c|c|c|c|c|c|c|}
\hline \multicolumn{4}{|c|}{ Medellín7. Binomial step-up/down. 3/31/2014 } & \multicolumn{4}{|c|}{ Grupo Funcionalidad. Alcance: 50} \\
\hline \multicolumn{2}{|c|}{ Existencia de par funcional } & Elisión & Peso & Sustitución & Peso & Total & $\%$ \\
\hline \multirow[t]{2}{*}{ Existe par funcional } & $\mathrm{N}$ & 472 & \multirow[t]{2}{*}{.96} & 18 & \multirow[t]{2}{*}{.04} & \multirow[t]{2}{*}{490} & \multirow[t]{2}{*}{5.1} \\
\hline & $\%$ & 96.3 & & 3.7 & & & \\
\hline \multirow[t]{2}{*}{ No existe par funcional } & $\mathrm{N}$ & 4220 & \multirow[t]{2}{*}{.46} & 4887 & \multirow[t]{2}{*}{.54} & \multirow[t]{2}{*}{9107} & \multirow[t]{2}{*}{94.9} \\
\hline & $\%$ & 46.3 & & 53.7 & & & \\
\hline \multirow[t]{2}{*}{ TOTAL } & $\mathrm{N}$ & \multicolumn{2}{|l|}{4692} & \multicolumn{2}{|l|}{4905} & \multirow{2}{*}{\multicolumn{2}{|c|}{9597}} \\
\hline & $\%$ & 48.9 & & 51.1 & & & \\
\hline \multicolumn{8}{|c|}{ Input 0.489 Log likelihood $=-6649.770$} \\
\hline
\end{tabular}

Cuadro 3. Funcionalidad. Pesos y distribución

En el cuadro 3 vemos que una gran mayoría de elisiones y sustituciones (94.9\%) se presenta cuando la palabra en la que ocurren los procesos no tiene par funcional que pueda llevar a ambigüedades del contenido del mensaje. Sin embargo, en una pequeña proporción, existiendo la posibilidad de generar ambigüedades por la modificación y coincidencia con otra palabra del léxico, se presenta una fuerte influencia (.96) a modificar el lexema a través del proceso de elisión. En otras palabras, cuando no existe la posibilidad de ambigüedad, se recurre más a los procesos de elisión y sustitución; pero aún con la existencia de pares funcionales, la preferencia por las elisiones es alta en relación con el uso de las sustituciones. En consecuencia, al darse la opción de elidir o sustituir un sonido en una palabra se prefiere hacerlo sobre aquellas que no conlleven ambigüedades o interrupciones en la transmisión de la información.

Para Klamer (2005: 131) la estructuración de la comunicación lingüística en su relación con los aspectos de la economía del lenguaje puede verse desde dos perspectivas: la del hablante, al mostrarse una tendencia en la economía de la producción, y la del oyente, al mostrarse una tendencia a la economía en el proce- 
samiento. Si atendemos a la economía del hablante, se tiende al menor esfuerzo y se reducen los elementos lingüísticos en contraste. Si atendemos a la economía del oyente, se tiende a la mayor distinción y se reducen los elementos lingüísticos idénticos.

Podemos inferir entonces que en esta variedad, en general, se coopera con la comprensión del mensaje ya que se aplican procesos fonológicos sobre palabras que en su mayoría no conducen a la ambigüedad porque no tienen pares funcionales si se modifican las formas canónicas, a pesar de reconocerse que la variación fonológicamente regular es aceptada por acomodación perceptual e inferencia (Gaskell y Marslen-Wilson, 1996: 144).

Sin embargo, como se constata que la existencia de pares funcionales, en un grupo reducido de palabras, contribuye mucho más con la presencia de la elisión que de la sustitución, se muestra en la formación del léxico en esta variedad de habla una ligera tendencia a la economía de la producción. Las elisiones son parte del proceso de debilitamiento por el cual la fuerza articulatoria manifiesta reducción total (se reduce un sonido a cero) para ciertas posiciones fonológicas, lo que implica menor esfuerzo por parte del hablante (Kuzmanovic, 2005: 649; Lass, 1984: 187).

\section{El efecto del Entorno segmental de palabra}

\begin{tabular}{|c|c|c|c|c|c|c|c|}
\hline \multicolumn{4}{|c|}{ Medellín7. Binomial step-up/down. 3/31/2014. } & \multicolumn{4}{|c|}{$\begin{array}{l}\text { Grupo Entorno segmental de } \\
\text { palabra. Alcance: } 48\end{array}$} \\
\hline \multicolumn{2}{|c|}{ Presencia del mismo segmento } & Elisión & Peso & Sustitución & Peso & Total & $\%$ \\
\hline \multirow[t]{2}{*}{ No hay otro } & $\mathrm{N}$ & 3416 & \multirow[t]{2}{*}{.44} & 4612 & \multirow[t]{2}{*}{.56} & \multirow[t]{2}{*}{8028} & \multirow[t]{2}{*}{83.7} \\
\hline & $\%$ & 42.6 & & 57.4 & & & \\
\hline \multirow[t]{2}{*}{ Hay otro canónico } & $\mathrm{N}$ & 1234 & \multirow[t]{2}{*}{.81} & 208 & \multirow[t]{2}{*}{.19} & \multirow[t]{2}{*}{1442} & \multirow[t]{2}{*}{15.0} \\
\hline & $\%$ & 85.6 & & 14.4 & & & \\
\hline \multirow[t]{2}{*}{ Hay otro modificado } & $\mathrm{N}$ & 42 & \multirow[t]{2}{*}{.33} & 85 & \multirow[t]{2}{*}{.67} & \multirow[t]{2}{*}{127} & \multirow[t]{2}{*}{1.3} \\
\hline & $\%$ & 33.1 & & 66.9 & & & \\
\hline \multirow[t]{2}{*}{ TOTAL } & $\mathrm{N}$ & \multicolumn{2}{|l|}{4692} & \multicolumn{2}{|l|}{4905} & \multirow{2}{*}{\multicolumn{2}{|c|}{9597}} \\
\hline & $\%$ & 48.9 & & 51.1 & & & \\
\hline
\end{tabular}

Cuadro 4. Entorno segmental de palabra. Pesos y distribución 
Se observa en el cuadro 4 una altísima frecuencia (83.7\%) de elisiones y sustituciones cuando no hay presencia de un segmento homólogo en la palabra; es decir, no hay en la misma palabra un fonema idéntico, por ejemplo, en la sustitución [ $\mathrm{r}]$

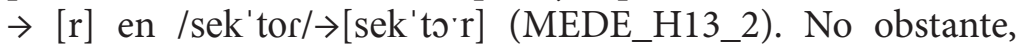
cuando hay presencia de un segmento homólogo canónico se favorece más la elisión (.81), como en el caso $/ \mathrm{d} / \rightarrow[\varnothing]$, / 'donde/ $\rightarrow$ ['õ'nde] (MEDE_H13_2). Y, aunque con muy pocos casos $(\mathrm{N}=127)$, la presencia de un segmento homólogo modificado contribuye más con la sustitución; como se presenta para $/ \mathrm{s} / \rightarrow[\mathrm{h}]$ en [nõ'ho'tro] por /no'sotros/ (MEDE_H33_3).

En términos de la teoría de la producción del habla y en relación con el cambio lingüístico surgido de los errores de habla, se ha constatado que cuando hay segmentos cercanos con rasgos idénticos, el patrón de inhibición en la planeación motora elimina la repetición, conservando el segmento más importante (que ejerce influencia sobre el que lo antecede o que aparece en la posición más fuerte) (Garrett y Johnson, 2013: 23). Este hecho explica la razón por la cual la presencia de un segmento canónico favorece la elisión (.81). Para el caso de la favorabilidad de la sustitución como influenciada por la presencia de otro segmento homólogo modificado parece estar operando, en los pocos casos de aparición $(\mathrm{N}=127)$, el patrón de mezcla en la planeación motora que tiende a generar más procesos de asimilación por anticipación que de preservación (Garrett y Johnson, 2013: 13). Se trata en ambos casos (elisión y sustitución) de la variación fonológica que surge de la presencia de segmentos idénticos en la estructura léxica.

La relación cruzada entre el Entorno segmental de palabra y el Acento, presentada en el cuadro 5, muestra que la presencia del segmento en la palabra y en la sílaba tónica son dos factores que favorecen la sustitución. En sílaba tónica aumentan las sustituciones a medida que aparecen segmentos homólogos y se realizan aún más sustituciones si el segmento homólogo está modificado (cuadro 5).

Otro tanto sucede con el cruce entre el Entorno segmental de palabra y la Estructura morfemática que se registra en el cuadro 6. Ante la presencia de otro segmento modificado en la 
palabra se incrementa la aparición de sustituciones en los morfemas gramaticales. El cruce con posición silábica (ataque/coda) no mostró patrón explicativo.

\begin{tabular}{|l|l|l|l|}
\hline $\begin{array}{l}\text { Acento } \\
\text { Entorno segmental de palabra }\end{array}$ & Precede & Sigue & Tónica \\
\hline No hay otro & E 46 & E 88 & E 25 \\
& S 54 & S 12 & S 39 \\
\hline \multirow{2}{*}{ Hay canónico } & E 37 & E 83 & E 29 \\
& S 63 & S 17 & S 71 \\
\hline Hay modificado & E 42 & E 81 & E 18 \\
& S 58 & S 19 & S 82 \\
\hline
\end{tabular}

Cuadro 5. Porcentajes de las variables Entorno segmental de palabra y acento

\begin{tabular}{|l|l|l|}
\hline $\begin{array}{l}\text { Estructura morfemática } \\
\text { Entorno segmental de palabra }\end{array}$ & Morfema léxico & $\begin{array}{l}\text { Morfema } \\
\text { gramatical }\end{array}$ \\
\hline No hay otro & E 38 & E 61 \\
S 62 39
\end{tabular}

Cuadro 6. Porcentajes de las variables Entorno segmental de palabra y estructura morfemática

\section{El efecto del Número de sílabas}

De acuerdo con el número de sílabas de la palabra en la que se presenta el fenómeno, a mayor número de sílabas se favorece la elisión (.74 y .59), mientras que para la sustitución contribuye más un menor número de sílabas (.63 y .57), tal como se observa en el cuadro 7. 
Se referencia a menudo en la literatura que en palabras polisilábicas hay mayor posibilidad de elisión de elementos de la sílaba no acentuada, especialmente en conversaciones a velocidad normal (Crystal, 2000: 195). Es claro entonces que el grupo

\begin{tabular}{|c|c|c|c|c|c|c|c|}
\hline \multicolumn{4}{|c|}{$\begin{array}{l}\text { Medellín7. Binomial step-up/down. } \\
\text { 3/31/2014 }\end{array}$} & \multicolumn{4}{|c|}{$\begin{array}{l}\text { Grupo Número de sílabas. } \\
\text { Alcance: } 37\end{array}$} \\
\hline \multicolumn{2}{|c|}{$\begin{array}{l}\text { Tipo de palabra } \\
\text { por número de } \\
\text { sílabas }\end{array}$} & Elisión & Peso & Sustitución & Peso & Total & $\%$ \\
\hline \multirow[t]{2}{*}{ Bisílabas } & $\mathrm{N}$ & 1419 & \multirow[t]{2}{*}{.37} & 2476 & \multirow[t]{2}{*}{.63} & \multirow[t]{2}{*}{3895} & \multirow[t]{2}{*}{40.6} \\
\hline & $\%$ & 36.4 & & 63.6 & & & \\
\hline \multirow[t]{2}{*}{ Trisílabas } & $\mathrm{N}$ & 1263 & \multirow[t]{2}{*}{.59} & 1349 & \multirow[t]{2}{*}{.41} & \multirow[t]{2}{*}{2612} & \multirow[t]{2}{*}{27.2} \\
\hline & $\%$ & 48.4 & & 51.6 & & & \\
\hline \multirow[t]{2}{*}{ Polisílabas } & $\mathrm{N}$ & 1058 & \multirow[t]{2}{*}{.74} & 437 & \multirow[t]{2}{*}{.26} & \multirow[t]{2}{*}{1495} & \multirow[t]{2}{*}{15.6} \\
\hline & $\%$ & 70.8 & & 29.2 & & & \\
\hline \multirow[t]{2}{*}{ Monosílabas } & $\mathrm{N}$ & 952 & \multirow[t]{2}{*}{.43} & 643 & \multirow[t]{2}{*}{.57} & \multirow[t]{2}{*}{1595} & \multirow[t]{2}{*}{16.6} \\
\hline & $\%$ & 59.7 & & 40.3 & & & \\
\hline \multirow[t]{2}{*}{ TOTAL } & $\mathrm{N}$ & \multicolumn{2}{|l|}{4692} & \multicolumn{2}{|l|}{4905} & \multirow{2}{*}{\multicolumn{2}{|c|}{9597}} \\
\hline & $\%$ & 48.9 & & 51.1 & & & \\
\hline
\end{tabular}

Cuadro 7. Número de sílabas. Pesos y porcentajes

factorial debe cruzarse en primer lugar con el acento y la fase de interacción para ser interpretado con mayor poder explicativo.

\begin{tabular}{|l|l|l|l|}
\hline $\begin{array}{l}\text { Número de sílabas } \\
\text { Acento }\end{array}$ & Bisílaba & Trisílaba & Polisílaba \\
\hline Precede & E 48 & E 64 & E84 \\
& S 52 & S 36 & S16 \\
\hline Tónica & E 30 & E 43 & E73 \\
\hline Sigue & S 70 & S 57 & S27 \\
\hline & E 19 & E 39 & E61 \\
\hline
\end{tabular}

Cuadro 8. Porcentajes de Número de sílabas y Acento 
El cuadro 8 presenta unas relaciones paralelas del comportamiento del uso de los procesos en estudio: a mayor número de sílabas, mayor proporción de elisiones, en mayor medida para las pretónicas, seguidas por las tónicas y en menor proporción para las postónicas. Esto muestra que el Número de sílabas contrarresta la influencia que pudiera tener la Estructura acentual ya que a medida que aumenta el número de sílabas aumenta la proporción de elisiones sin importar que éstas aparezcan en sílaba pretónica, tónica o postónica. Asimismo, se decrementa el número de sustituciones a medida que se va aumentando el número de sílabas, en proporciones menores desde lo pretónico hasta lo postónico.

El cruce entre Número de sílabas y Fase de interacción, expuesto en el cuadro 9, muestra que las elisiones y las sustituciones en el número de sílabas siguen un patrón similar al de su relación con el acento. En cada tipo de registro, a mayor número de sílabas, mayor proporción de elisiones, salvo para los monosílabos; de igual forma, a menor número de sílabas, mayor proporción de sustituciones, sin contar los monosílabos. No hay una gradación de lo formal a lo coloquial; se conservan proporciones similares en cada fase.

\begin{tabular}{|l|l|l|l|l|}
\hline $\begin{array}{l}\text { Número de sílabas } \\
\text { Fase de interacción }\end{array}$ & Monosílabo & Bisílaba & Trisílaba & Polisílaba \\
\hline Formal & E 63 & E 39 & E 49 & E 74 \\
& S 37 & S 61 & S 51 & S 26 \\
\hline$+/$ - formal & E 59 & E 34 & E 46 & E 67 \\
& S 41 & S 66 & S 54 & S 33 \\
\hline$+/$ coloquial & E 58 & E 36 & E 50 & E 71 \\
& S 42 & S 64 & S 50 & S 29 \\
\hline
\end{tabular}

Cuadro 9. Tabla cruzada. Porcentajes de Número de sílabas y Fase de la interacción

Este resultado nos acerca al reconocimiento de las unidades de procesamiento del lenguaje más allá del segmento, como la sílaba y el acento, en su papel de guías de la estructuración y la manifestación del habla. Vemos cómo la sílaba es una entidad funcionalmente relevante en la producción del habla en 
español, como se ha evidenciado para el estudio de los errores de habla (Gutiérrez, Palma y Santiago, 2003).

\section{El efecto de la Homofonía}

El uso de los procesos fonológicos es mucho mayor en las palabras que no tienen homófonos en el léxico (73.5\%), como se muestra en el cuadro 10. Este factor favorece la aparición de las sustituciones (.58). De otra parte, la existencia de homófonos entre categorías gramaticales distintas a nombre, verbo y adjetivo contribuye con el proceso de elisión (.77).

\begin{tabular}{|c|c|c|c|c|c|c|c|}
\hline \multicolumn{4}{|c|}{$\begin{array}{l}\text { Medellín7. Binomial step-up/down. } \\
\text { 3/31/2014. }\end{array}$} & \multicolumn{4}{|c|}{ Grupo Homofonía. Alcance: 35} \\
\hline \multicolumn{2}{|l|}{ Existencia de homófono } & Elisión & Peso & Sustitución & Peso & Total & $\%$ \\
\hline \multirow{2}{*}{ No hay homofonía } & $\mathrm{N}$ & 2871 & \multirow[t]{2}{*}{.42} & 4178 & \multirow[t]{2}{*}{.58} & \multirow[t]{2}{*}{7049} & \multirow[t]{2}{*}{73.5} \\
\hline & $\%$ & 40.7 & & 59.3 & & & \\
\hline \multirow[t]{2}{*}{ Homófono $\mathrm{N}$ - V - Adj. } & $\mathrm{N}$ & 462 & \multirow[t]{2}{*}{.52} & 385 & \multirow[t]{2}{*}{.48} & \multirow[t]{2}{*}{847} & \multirow[t]{2}{*}{8.8} \\
\hline & $\%$ & 54.5 & & 45.5 & & & \\
\hline \multirow[t]{2}{*}{ Homófono otras cat. } & $\mathrm{N}$ & 1359 & \multirow[t]{2}{*}{.77} & 342 & \multirow[t]{2}{*}{.23} & \multirow[t]{2}{*}{1701} & \multirow[t]{2}{*}{17.7} \\
\hline & $\%$ & 79.9 & & 20.1 & & & \\
\hline \multirow[t]{2}{*}{ TOTAL } & $\mathrm{N}$ & \multicolumn{2}{|l|}{4692} & \multicolumn{2}{|l|}{4905} & \multirow{2}{*}{\multicolumn{2}{|c|}{9597}} \\
\hline & $\%$ & 48.9 & & 51.1 & & & \\
\hline \multicolumn{8}{|c|}{ Input 0.489 Log likelihood $=-6649.770$} \\
\hline
\end{tabular}

Cuadro 10. Homofonía. Pesos y porcentajes

Además, al revisar el cruce de la Homofonía con la Difusión léxica, en el cuadro 11, se observó que en el léxico de baja difusión es donde se incrementa la proporción de las elisiones cuando en el léxico hay homofonía con otras categorías léxicas.

\begin{tabular}{|l|l|l|}
\hline $\begin{array}{l}\text { Difusión léxica } \\
\text { Homofonía }\end{array}$ & Alta & Baja \\
\hline No hay homófono & E 42 & E 37 \\
S 63 58 \\
\hline Homofonía NVAdj. & E 55 & E 50 \\
S 50 5 \\
\hline Homofonía otras categorías léxicas & E 70 & E 96 \\
\hline
\end{tabular}

Cuadro 11. Porcentajes de Homofonía y Difusión léxica 
Tenemos entonces que, en general, se aplican procesos en palabras que no tienen homófonos. En este sentido, existe muy buena cooperación con la comprensión del mensaje ya que se ha constatado que la existencia de homófonos en las lenguas incrementan la dificultad en el procesamiento del lenguaje (Klamer, 2005: 130): lo que prefieren las lenguas es enlazar significados diferentes a morfemas diferentes, es decir, establecer una relación uno a uno entre forma y función. La preferencia de los hablantes por aplicar elisiones y sustituciones sobre palabras que no tienen homófonos va en la vía de la cooperación en el procesamiento.

La homofonía entre categorías N, V y Adj., al pertenecer estas a los morfemas léxicos con mayor carga en el mensaje incrementa en mayor medida el grado de dificultad del procesamiento. La proporción de procesos en palabras que incurren en este factor es mínima y no hay influencia en la aplicación de elisiones y sustituciones. Puede verse también este aspecto como una forma de cooperación con la comprensión del mensaje. Para Klamer (2005: 130) el seguimiento de la restricción sobre las formas homófonas puede verse desde las dos perspectivas ya mencionadas: la del hablante y la del oyente y su relación con la producción y el procesamiento, respectivamente. El hecho de que se presenten modificaciones en las palabras cuando no existe dificultad en el procesamiento al no haber homofonía o muy poca presencia de procesos en palabras con homófonos de categorías N, V y Adj., muestra la tendencia de la variedad a no incurrir en el incremento de la dificultad en el procesamiento y cooperar con la economía desde el punto de vista del oyente.

De otra parte, la presencia de homofonía entre categorías distintas a nombre, verbo y adjetivos, aunque en una proporción baja, contribuye más con el incremento de elisiones que de sustituciones, sobre todo en léxico de baja difusión. De este hecho se puede inferir que en esta variedad de habla existe también una tendencia a la economía desde el punto de vista del hablante, que apela a la economía de la producción del habla (Klamer, 2005: 131). Nuevamente, se constata un menor esfuerzo articulatorio por parte del hablante.

Vemos que entre las categorías que mayor influencia tuvieron para la aplicación de procesos de elisión y sustitución se 
encuentran dos variables léxico-semánticas: la funcionalidad y la homofonía (con alcances de 50 - el máximo- - 35, respectivamente). Estas dos categorías muestran un patrón de procedimiento relacionado con el procesamiento del lenguaje y la producción del habla. Los procesos de elisión y sustitución se aplican en su mayoría sobre formas léxicas que no dificulten el procesamiento. Cuando se aplican con léxico que ya tiene una dificultad en su procesamiento, coincidente en menor proporción, muy pocos casos, se apela a la economía de la producción a través de la reducción total de la fuerza articulatoria manifiesta en el incremento de las elisiones.

\section{El efecto de la Homorganicidad ${ }^{10}$}

Este grupo factorial es el quinto que más influye para la selección de los procesos, con un alcance de 30 . Obsérvese el cuadro 12.

\begin{tabular}{|c|c|c|c|c|c|c|c|}
\hline \multicolumn{4}{|c|}{$\begin{array}{l}\text { Medellín7. Binomial step-up/down. } \\
\text { 3/31/2014 }\end{array}$} & $\begin{array}{l}\text { Grupo } \\
\text { Alcance: } 30\end{array}$ & \multicolumn{3}{|c|}{ Homorganicidad. } \\
\hline \multicolumn{2}{|l|}{$\begin{array}{l}\text { Existencia de segmento } \\
\text { homorgánico }\end{array}$} & Elisión & Peso & Sustitución & Peso & Total & $\%$ \\
\hline \multirow{2}{*}{$\begin{array}{l}\text { No hay segmento } \\
\text { homorgánico }\end{array}$} & $\mathrm{N}$ & 3599 & \multirow{2}{*}{$\begin{array}{l}.62 \\
38.4\end{array}$} & 2248 & \multirow[t]{2}{*}{.38} & \multirow[t]{2}{*}{5847} & \multirow[t]{2}{*}{60.9} \\
\hline & $\%$ & 61.6 & & & & & \\
\hline \multirow[t]{2}{*}{ Hay homorganicidad } & $\mathrm{N}$ & 1093 & \multirow{2}{*}{$\begin{array}{l}.32 \\
70.9 \\
\end{array}$} & 2657 & \multirow[t]{2}{*}{.68} & \multirow[t]{2}{*}{3750} & \multirow[t]{2}{*}{39.1} \\
\hline & $\%$ & 29.1 & & & & & \\
\hline \multirow[t]{2}{*}{ TOTAL } & $\mathrm{N}$ & \multicolumn{2}{|l|}{4692} & \multicolumn{2}{|l|}{4905} & \multirow{2}{*}{\multicolumn{2}{|c|}{9597}} \\
\hline & $\%$ & 48.9 & & 51.1 & & & \\
\hline \multicolumn{8}{|c|}{ Input 0.489 Log likelihood $=-6649.770$} \\
\hline
\end{tabular}

Cuadro 12. Homorganicidad. Pesos y porcentajes

\footnotetext{
${ }^{10}$ En este trabajo, la Homorganicidad se codificó para los segmentos en adyacencia, en contextos pre y postsegmentales, que coinciden únicamente en el rasgo de punto de articulación. Así, se incluyeron los casos de yeísmo ante vocales palatales, en ejemplos como la pronunciación tan frecuente de Medellín como [mee'jin]. Este fenómeno explica la influencia de la homorganicidad en el porcentaje y el peso de las sustituciones.
} 
El peso Goldvarb del factor 'sin homorganicidad' (.62) que favorece la presencia del proceso de elisión contrasta con el peso probabilístico del factor 'existencia de homorganicidad' que contribuye con la aparición del fenómeno de sustitución. Esto indica que frente a la coocurrencia de segmentos homorgánicos o la adyacencia de segmentos coincidentes en rasgos homorgánicos se sigue una restricción fonotáctica que desfavorece la combinación de elementos homorgánicos en contacto a través principalmente de la sustitución.

Para los casos en que hay elisiones, aunque en poca proporción y sin fuerza de influencia, se destaca que si se relaciona la Homorganicidad con la Posición silábica (cuadro 13). Vemos cómo se incrementa el uso de la elisión en las sílabas trabadas por consonante cuando hay elementos adyacentes homorgánicos, por ejemplo en enojá(r)sele, que coinciden en la alveolaridad. La elisión de segmento homorgánico en coda se corrobora aún más cuando cruzamos el factor Homorganicidad con el grupo factorial Contexto segmental. El cuadro 14 nos muestra la manera casi categórica en que el fenómeno de elisión consonántica está ligado a la presencia de otro segmento consonántico homorgánico.

\begin{tabular}{|l|l|l|}
\hline $\begin{array}{l}\text { Posición silábica } \\
\text { Homorganicidad }\end{array}$ & Ataque & Coda \\
\hline $\begin{array}{l}\text { No hay } \\
\text { homorganicidad }\end{array}$ & $\begin{array}{l}\text { E 78 } \\
\text { S 22 }\end{array}$ & $\begin{array}{l}\text { E 50 } \\
\text { S 50 }\end{array}$ \\
\hline $\begin{array}{l}\text { Hay } \\
\text { homorganicidad }\end{array}$ & E 4 & E 77 \\
S 96 & S 23 \\
\hline
\end{tabular}

Cuadro 13. Porcentajes de Homorganicidad y Posición silábica

\begin{tabular}{|l|l|l|}
\hline $\begin{array}{l}\text { Contexto segmental } \\
\text { Homorganicidad }\end{array}$ & V-V & V-C \\
\hline $\begin{array}{l}\text { No hay } \\
\text { homorganicidad }\end{array}$ & $\begin{array}{l}\text { E 74 } \\
\text { S 26 }\end{array}$ & E 49 \\
\hline S 52 \\
\hline Hay homorganicidad & E 1 & E 79 \\
S 99 & S 21 \\
\hline
\end{tabular}

Cuadro 14. Porcentajes de Homorganicidad y Contexto segmental

Ahora bien, aunque el favorecimiento de la elisión está dado por el peso de la ausencia de Homorganicidad, en los casos en que se presenta elisión cuando hay segmento homorgánico, en especial en coda silábica y ante consonante, la elisión se incrementa en buena medida. En este sentido, vemos cómo la estructura silábica entra de nuevo a jugar un papel relevante. Sin embargo, de acuerdo con Gibson (2010: i-ii), son los principios 
de buena formación de ataques los que dominan la estructura silábica del español mientras que las codas se toleran pasivamente. Según este autor, el resultado natural de esa afirmación es que la inclusión de restricciones especiales que gobiernen la buena formación de codas es superflua y en cierta manera redundante. La estructura silábica establece unas posiciones fuertes y otras débiles, que efectivamente influyen en la aparición de procesos, más aún si hay homorganicidad.

Observamos hasta aquí que entre las categorías de mayor influencia para aplicación de procesos de elisión y sustitución se encuentran tres variables de nivel fonológico. El Entorno segmental de palabra y la Homorganicidad relacionan su influencia a través de un patrón de estrategia para evitar la similitud: si hay segmentos idénticos no adyacentes se favorece el proceso de elisión, mientras que si hay segmentos no idénticos adyacentes se favorece el proceso de sustitución. Se agrega a esto la importancia de la sílaba en la especificación fonológica: el número de sílabas contribuye en el incremento de elisiones; de igual forma, hay aumento de las elisiones para la posición de coda en casos de ausencia de homorganicidad.

\section{El efecto del Acento}

\begin{tabular}{|c|c|c|c|c|c|c|c|}
\hline \multicolumn{4}{|c|}{$\begin{array}{l}\text { Medellín7. Binomial step-up/down. } \\
\text { 3/31/2014 }\end{array}$} & \multicolumn{4}{|c|}{$\begin{array}{l}\text { Grupo Acento. } \\
\text { Alcance: } 28\end{array}$} \\
\hline \multicolumn{2}{|c|}{ Posición de la sílaba tónica } & Elisión & Peso & Sustitución & Peso & Total & $\%$ \\
\hline \multirow[t]{2}{*}{ Precede al segmento } & $\mathrm{N}$ & 1546 & \multirow[t]{2}{*}{.70} & 1123 & \multirow[t]{2}{*}{.30} & \multirow[t]{2}{*}{2669} & \multirow[t]{2}{*}{27.8} \\
\hline & $\%$ & 57.9 & & 42.1 & & & \\
\hline \multirow[t]{2}{*}{ Sigue al segmento } & $\mathrm{N}$ & 670 & \multirow[t]{2}{*}{.42} & 844 & \multirow[t]{2}{*}{.58} & \multirow[t]{2}{*}{1514} & \multirow[t]{2}{*}{15.8} \\
\hline & $\%$ & 44.3 & & 55.7 & & & \\
\hline \multirow[t]{2}{*}{ En sílaba tónica } & $\mathrm{N}$ & 2476 & \multirow[t]{2}{*}{.42} & 2938 & \multirow[t]{2}{*}{.58} & \multirow[t]{2}{*}{5414} & \multirow[t]{2}{*}{56.4} \\
\hline & $\%$ & 45.7 & & 54.3 & & & \\
\hline \multirow[t]{2}{*}{ TOTAL } & $\mathrm{N}$ & \multicolumn{2}{|l|}{4692} & \multicolumn{2}{|l|}{4905} & \multirow{2}{*}{\multicolumn{2}{|c|}{9597}} \\
\hline & $\%$ & 48.9 & & 51.1 & & & \\
\hline \multicolumn{8}{|c|}{ Input 0.489 Log likelihood $=-6649.770$} \\
\hline
\end{tabular}

Cuadro 15. Grupo factorial Acento. Pesos y porcentajes 
Aunque no tuvo un alcance del mismo nivel que los factores anteriores, se destaca este factor dada su relevancia para la conformación prosódica del léxico y las correlaciones que presenta. Los resultados presentados en el cuadro 15 y relacionados con la posición del fenómeno en estudio muestran un favorecimiento de las sílabas postónicas para la probabilidad de elisiones (.70). Las sílabas pretónicas y las tónicas contribuyen con el proceso de sustitución (.58), presentándose más casos en las sílabas tónicas $(\mathrm{N}=2938)$. Concuerdan estas evidencias con muchos estudios que presentan a las sílabas postónicas como una estructura débil frente a las sílabas pretónicas y tónicas como estructuras fuertes, generadas por las posiciones prominentes prosódicamente en la cadena fónica (Lloret y Jiménez, 2008: 55). En consecuencia, se refuerza la idea de que las estructuras fuertes albergan segmentos que son más resistentes al cambio y más estables: en este caso se dispara la variación por sustitución; mientras que las estructuras débiles son más propensas al cambio, menos estables: en este caso se dispara la elisión. Podría bien interpretarse que los procesos de sustitución implican modificaciones menos radicales que los procesos de elisión ya que al reemplazar un fonema por otro se mantiene la posición (por ejemplo, es menos radical realizar la /s/ como una aspiración que eliminarla totalmente).

Responde así esta situación al seguimiento de la restricción de fidelidad posicional ocasionada por la prominencia prosódica en el componente fonológico de la gramática que dota a las sílabas tónicas y pretónicas de características acústicas y articulatorias que las destacan (Beckman, 1998: 185). Esta restricción opera sobre otras estructuras tales como la posición silábica y la estructura acentual, aunque, como ya vimos en la tabulación cruzada de el cuadro 8 entre Número de sílabas y Estructura acentual, el primer factor contrarresta el efecto del segundo: a mayor número de sílabas, mayor tendencia a la elisión en cualquier posibilidad de estructura acentual; a menor número de sílabas, mayor tendencia a la sustitución en cualquier posibilidad de estructura acentual. 


\begin{tabular}{|l|l|l|l|}
\hline $\begin{array}{l}\text { Fase de interacción } \\
\text { Acento }\end{array}$ & Formal & +/-formal & +/- informal \\
\hline Precede & E 69 & E 53 & E 53 \\
& S 31 & S 47 & S 47 \\
\hline Sigue & E 48 & E 45 & E 40 \\
& S 52 & S 55 & S 60 \\
\hline Tónica & E 44 & E 44 & E 48 \\
& S 56 & S 56 & S 52 \\
\hline
\end{tabular}

Cuadro 16. Porcentajes de Acento y Fase de interacción

Ahora bien, el cuadro 16 muestra la correlación entre el acento y la fase de interacción en el uso: hay un ligero incremento de elisiones en las sílabas postónicas en situación de inicio de la entrevista y de igual manera un incremento de las sustituciones en sílabas pretónicas en situación de final de entrevista.

Esta correlación de resultados muestra un seguimiento de patrones que relacionan los fenómenos de elisión y sustitución con la longitud silábica y la posición del acento léxico. Se puede afirmar entonces que el número de sílabas y el acento condicionan la información fonológica segmental en la producción léxica, así: a mayor número de sílabas, mayor proporción de elisiones, en mayor medida para las pretónicas, seguidas por las tónicas y en menor proporción para las postónicas. Sin contar los monosílabos, a mayor número de sílabas, mayor proporción de elisiones, a menor número de sílabas, mayor proporción de sustituciones. Todo ello proporcional tanto en registro formal como en coloquial.

Otros resultados relacionados con grupos factoriales de alcance más bajo dan cuenta de la corroboración de tendencias de la evolución de la lengua: hay posiciones privilegiadas y prominentes como el ataque, la raíz, sílabas iniciales y finales, que favorecen la preservación de la canonicidad, así, las elisiones se presentan más en codas, en sílabas átonas y en morfemas gramaticales (Beckman, 1998).

Una característica destacable es que los resultados de la relación entre los procesos de elisión y sustitución y la pausa demuestran que al inicio y al final de los grupos fónicos (cadena 
fónica entre pausas) los hablantes de esta variedad pronuncian los segmentos con un máximo de correspondencia; la pausa no afecta los fonemas adyacentes. Es un hecho que caracteriza a esta variedad de habla: los procesos de elisión y sustitución se concentran al interior de los grupos fónicos y no en sus límites.

\section{Conclusiones}

El análisis cualitativo y cuantitativo de los fenómenos de elisión y la sustitución como fenómenos comunes y sistemáticos de variación fonológica en el habla de Medellín me permite concluir que la relativa cercanía frecuencial de los dos fenómenos controvierte la idea de que en los contextos semiespontáneos prevalecen los fenómenos de simplificación por encima de los de sustitución.

La significatividad, el peso y el alcance de los factores en el análisis de regresión mostraron que hay una relevancia de los factores internos sobre los externos, lo que podría ir en contravía a la idea de que cualquier variación fonológica va de la mano de la variación social.

El cálculo probabilístico y el análisis de regresión múltiple mostraron que las variables Funcionalidad, Entorno segmental de palabra, Número de sílabas, Homofonía y Homorganicidad fueron los factores que más contribuyeron para que se presenten procesos de elisión y sustitución en el habla de Medellín.

Entre las categorías que mayor influencia tuvieron para la aplicación de procesos de elisión y sustitución se encuentran dos variables léxico-semánticas: la funcionalidad y la homofonía. Estas dos categorías muestran un patrón de procedimiento relacionado con el procesamiento del lenguaje y la producción del habla. Los procesos se aplican en su mayoría sobre formas léxicas que no dificulten el procesamiento. Cuando se aplican con léxico que ya tiene una dificultad en su procesamiento, coincidente en menor proporción, muy pocos casos, se apela a la economía de la producción a través de la reducción total de la fuerza articulatoria manifiesta en el incremento de las elisiones. 
Entre las categorías de mayor influencia para aplicación de procesos de elisión y sustitución se encuentran tres variables de nivel fonológico. El Entorno segmental de palabra y la Homorganicidad relacionan su influencia a través de un patrón de estrategia en la producción del habla que evita la similitud: si hay segmentos idénticos no adyacentes se favorece el proceso de elisión, mientras que si hay segmentos no idénticos adyacentes se favorece el proceso de sustitución. Se agrega a esto la importancia de la sílaba en la especificación fonológica: el número de sílabas contribuye en el incremento de elisiones; de igual forma, hay aumento de las elisiones para la posición de coda en casos de ausencia de homorganicidad.

Se explicó la relación multicausal entre aspectos semánticos como la funcionalidad y la homofonía y aspectos de nivel fonológico como el entorno segmental de palabra, el número de sílabas, la homorganicidad y el acento como variables internas que guían el procesamiento del lenguaje para la estructuración léxica en la posibilidad variable de elicitar o sustituir una posición fonológica en la cadena fónica. Los patrones interrelacionados con factores estilísticos responden a restricciones del orden de la transparencia semántica y la evasión de la similitud en mayor medida para colaborar con la comprensión del mensaje. En este sentido, la variedad medellinense de habla, en general, coopera con la comprensión del mensaje.

Finalmente, de acuerdo con el estudio realizado y sus conclusiones, puedo esbozar una nueva definición del proceso fonológico como un fenómeno de procesamiento del lenguaje que permite relacionar divergencias entre la planeación en la estructura interna y la forma superficial de la cadena fónica. El proceso fonológico obedece a condicionamientos dados por las relaciones entre elementos y restricciones fonotácticas. Cada lengua, como producto social e histórico establece el sistema de preferencias que se usan en las comunidades y que marcan tendencias de uso en el habla. 


\section{Bibliografía}

Anderson, S. R. (1980), “Why Phonology isn't 'Natural”, Working Papers in Phonetics, 51, pp. 36-93.

Andrade Rodríguez, R., M. C. González Rátivay D. A. JARAMILLO YePES (2008), "La representatividad poblacional en el estudio sociolingüístico de Medellín”, Lenguaje, 36(2), pp. 527-549.

Battistella, E. L. (1996), The logic of markedness, Oxford, Oxford University Press.

Beckman, J. N. (1998), Positional Faithfulness, University of Massachussets, disponible en línea en http://roa.rutgers. edu/files/234-1297/234-1297-BECKMAN-3-0.PDF

Boersma, P. y D. Weenink (2014), Praat: doing Phonetics by Computer, disponible en línea en http://www.fon.hum. uva.nl/praat/

BRIz, A. (2010), "Lo coloquial y lo formal: el eje de la variación lingüística”, en R. M. Castañer Martín y V. Lagüéns Gracia (eds.), De moneda nunca usada. Estudios filológicos dedicados a José M. ${ }^{a}$ Enguita Utrilla, Zaragoza: Instituto "Fernando el Católico", pp. 125-134, disponible en línea en http:// ifc.dpz.es/recursos/publicaciones/29/95/11briz.pdf

Chambers, J. K., P. Trudgill y N. Schilling-Estes (2004), The Handbook of Language Variation and Change, Oxford, Blackwell.

Crystal, D. (2000), Diccionario de lingüística y fonética, Barcelona, Octaedro.

Denes, G., C. Semenza y E. Magno Caldognetto (1999), "Phonological disorders in aphasia", en Handbook of Clinical and Experimental Neuropsychology, Hove, Psychology Press.

Garrett, A. y K. Johnson. (2013), "Phonetic bias in sound change", en A. C. L. Yu (ed.), Origins of sound change: Approaches to phonologization, Oxford, Oxford University Press, pp. 51-97.

Gaskell, M. G. y W. D. Marslen-Wilson (1996), "Phonological variation and inference in lexical access", Journal of Ex- 
perimental Psychology. Human Perception and Performance, 22(1), pp. 144-158, disponible en línea en http://www. ncbi.nlm.nih.gov/pubmed/8742258

Gibson, M. A. (2010), Fonología del español: enfoque desde la Teoría de Optimidad, Madrid, Universidad Complutense de Madrid.

Goldrick, M. (2014), "Phonological processing: the Retrieval and Encoding the Word Form Information in Speech Production", en M. Goldrick, V. Ferreira y M. Miozzo (eds.), The Oxford Handbook of Language Productio, Oxford, Oxford University Press, pp. 228-244.

Gómez Molina, J. R., A. M. Ávila y M. Vida (2007), "Proyecto coordinado 'Estudio sociolingüístico del español de Las Palmas, Lleida, Granada, Madrid-Alcalá, Málaga y Valencia", en Actas del Congreso Internacional "Análisis del Dicurso Oral". Universidad de Almería, 23-25 de noviembre de 2005. Homenaje a J.J. de Bustos Tovar, Madrid, Arco Libros, pp. $203-219$.

González-Rátiva, M. C. (Coord.) (2008), Corpus lingüístico de Medellín, disponible en línea en http://comunicaciones. udea.edu.co/corpuslinguistico/

González-Rátiva, M. C. y R. Grajales Alzate (2011), "La clase social en el Corpus Preseea-Medellín”, Lenguaje, 39(1), pp. 41-64.

Gutiérrez, N., A. Palma y J. Santiago (2003), “El papel de la sílaba y de la rima en producción del lenguaje: Evidencia desde los errores del habla en español", Psicológica, 24(1, enero-junio), pp. 57-78, disponible en línea en http:// www.redalyc.org/pdf/169/16924104.pdf

Hulst, H. van DER (2001), The Phonological Structure of Words: An Introduction, Cambridge, Cambridge University Press. Hurtado Cubillos, L. M. (2012), "La variable expresión de la impersonalidad en el español de los colombianos de Bogotá y Miami”, en R. J. File-Muriel y R. Orozco (eds.), Colombian Varieties of Spanish, Madrid, IberoamericanaVervuert, pp. 141-168.

Juncos Rabadán, O., R., Elosúa de Juan, A. Pereiro Rozas 
y M. Del C.Torres Maroño (1998), "Problemas de acceso léxico en la vejez. Bases para la intervención", Anales de Psicologia, 14(2), pp. 169-176, disponible en línea en http:// digitum.um.es/xmlui/handle/10201/10153

Klamer, M. (2005), "Explaining some Structural and Semantic Asymmetries in Morphological Typology", en G. Booij, E. Guevara, A. Ralli, S. Sgroi y S. Scalise (eds.), Morphology and Linguistic Typology, Bolonia, Università degli Studi di Bologna, pp. 127-142, disponible en línea en http://morbo. lingue.unibo.it $/ \mathrm{mmm} /$

Kuzmanovic, A. (2005), "La reducción de la fuerza articulatoria: un mecanismo de cambio universal y su importancia para la historia del español”, Interlingüística, 16(2), pp. 649656.

Labov, W. (1996), Principios del cambio lingüístico: factores internos, volumen 1, Madrid, Gredos.

Labov, W. (2006), Principios del cambio lingüístico: factores sociales, volumen 2, Madrid, Gredos.

Lass, R. (1984), Phonology: An Introduction to Basic Concepts, Cambridge, Cambridge University Press.

Levelt, W. J. M. (1999), "Models of word production”. Trends in Cognitive Sciences, 3(6), pp. 223-232.

Levelt, W. J. M., A. Roelofs y A. S. Meyer (1999), "A theory of lexical access in speech production", Behavioral and Brain Sciences, 22(1), pp. 1-38.

LEVelt, W. J. M. (2001), "Spoken word production: a theory of lexical access", Proceedings of the National Academy of Sciences of the United States of America, 98(23), pp. 13464113471.

Lloret, M. R. y J. JimÉnEz (2008), “Marcatge posicional i prominència en el vocalisme àton", Caplletra, 45, pp. 55-91.

Macionis, J. J. y K. Plummer (1999), Sociología, Madrid, Prentice Hall.

PRESEEA (2003), Metodología del "Proyecto para el estudio sociolingüístico del español de España y América” (PRESEEA), Alcalá, disponible en línea en http://preseea.linguas.net/ Portals/0/Metodologia/METODOLOGíA PRESEEA.pdf 
Sankoff, D., S. Tagliamonte y E. Smith (2005), Goldvarb X: A variable rule application for Macintosh and Windows, disponible en línea en http://individual.utoronto.ca/tagliamonte/goldvarb.html

Tagliamonte, S. A. (2006), Analysing sociolinguistic variation, Cambridge, Cambridge University Press.

Varela Ortega, S. (1984), "Lo 'natural' en fonología", Estudios de Lingüística Universidad de Alicante, 2, pp. 91119, disponible en línea en http://rua.ua.es/dspace/bitstream/10045/6655/1/ELUA_02_03.pdf 